\title{
3D Body Scanning and Healthcare Applications
}

Developed largely for the clothing industry, 3D body-surface scanners are transforming our ability to accurately measure and visualize a person's body size, shape, and skin-surface area. Advancements in 3D whole-body scanning seem to offer even greater potential for healthcare applications.

Philip Treleaven

and Jonathan Wells University College London

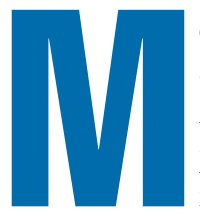

edical practitioners have traditionally measured the body's size and shape by hand to assess health status and guide treatment. More recently, computed-tomography $(\mathrm{CT})$ scanners have been able to produce 3D internal images of a patient's body. Now, 3D body-surface scanners are transforming the ability to accurately measure a person's body size, shape, and skin-surface area. Although developed primarily for the clothing industry, 3D scanners' low cost, noninvasive character, and ease of use make them appealing for widespread clinical applications and large-scale epidemiological surveys. ${ }^{1}$

\section{HIGHLY ACCURATE MAPS}

Whole-body scanners can capture highly accurate 3D body maps in 1 to 10 seconds. They let a computer automatically extract hundreds of measurements from a scan while eliminating manual measurement and transcription errors, allowing future remeasurement of scans, and greatly reducing the cost of anthropometric surveys. A body scan produces a point cloud, from which a computer extracts surface details, such as measurements associated with a body landmark as Figure 1 shows.

\section{Human-body metrics}

Technological advances in recent years have enabled diagnostic studies to reveal more detailed information about the body's internal structure. X-rays, magnetic resonance imaging (MRI), CT, and ultrasound have revolutionized the capacity to study physiology and anatomy in vivo and to aid in the diagnosis and monitoring of a multitude of disease states.

External measurements of the body are just as useful. Medical professionals widely use size and shape to assess nutritional status and developmental normality and to calculate the requirements of drug, radiotherapy, and chemotherapy dosages, as well as the production of prostheses. ${ }^{2}$

Three-dimensional scanners can have a major impact on medical research and practice. More detailed information about size and shape would transform the epidemiology of many diseases, replacing the current reliance on the body mass index 
(BMI), a proxy for quantifying many traits but an accurate marker of none. The capacity to obtain such information would considerably enhance physiological research, which requires regional indices of size to normalize and interpret data. Such information also could guide clinical practice in hospitals and community clinics.

\section{Body size and shape}

In addition to the 100 measurements the scanner can automatically extract, a clinician can also remeasure and examine the 3D digital body images and provide feedback to the patient.

Size and shape have implications for a variety of states of health or disease. Researchers are increasingly interested in investigating the proportions of the body that underlie total growth. For example, there's growing interest in body composition, in particular the central adiposity that characterizes obesity and is strongly linked to the risk of several lifestyle-related diseases.

\section{Skin-surface area}

The body's surface area is fundamentally related to several physiological processes, including water turnover and metabolic rate. Medical professionals often calculate body-surface area when prescribing certain drugs, dialysis rates, and similar treatments. Three-dimensional scanners image the entire body-surface area or individual limbs and body parts from a single scan.

The link between physiology and surface area derives from the association between nephron distribution and area. Surface area is also important when considering the administration of certain agents or effects that enter or meet the body through its surface. Burns and similar types of trauma damage the proportion of exposed skin, and treatment involves assessing the extent of the damaged tissue. Likewise, medical professionals calculate radiotherapy doses on the basis of the area exposed to limit toxicity.

\section{D visualization}

Three-dimensional scanners also provide visualization, which is highly important for clinician-patient interaction in cosmetic surgery.

While clinicians use measurements of body properties to assess risk and guide health management, patients use the same information in the context of other aspects of their lives, in particular their body image and sense of self-esteem.

In addition to providing simulation models to use in illustrating potential effects of treatment, visualization lets medical professionals demonstrate changes to the patient. Such models can potentially greatly improve the patient's understanding of treatment.

\section{D WHOLE-BODY SCANNING}

Research on building robust and accurate models to represent real human bodies began in the middle and

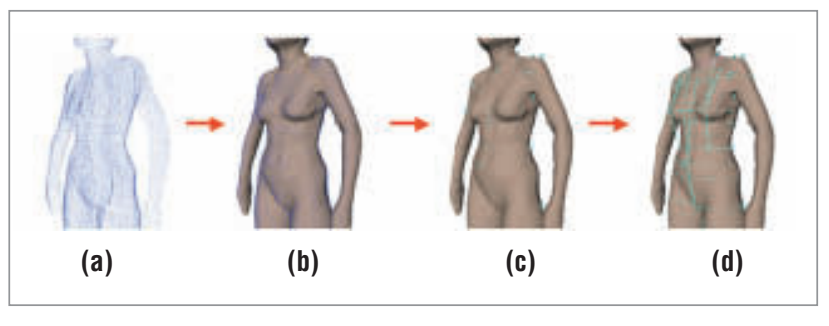

Figure 1. Virtual tape measure. Measurements can be extracted from (a) a point cloud, (b) surface reconstruction, (c) body landmarks, and (d) an electronic tape measure.

\begin{tabular}{lll}
\hline Table 1. Taxonomy of 3D body scanners. & \\
\hline Technique & Measurement & Visualization \\
\hline Photogrammetry & $\begin{array}{l}\text { Structured light } \\
\text { Moire fringe contouring }\end{array}$ & $\begin{array}{c}\text { Close-range } \\
\text { photogrammetry } \\
\end{array}$ \\
& Phase-measuring & $\begin{array}{c}\text { Digital surface } \\
\text { photogrammetry }\end{array}$ \\
\hline Laser & Laser scanners & \\
\hline Millimeter wave & Laser range scanners & \\
\hline & Radio waves & \\
\hline
\end{tabular}

late 1990s, when the first 3D-scanning devices emerged on the market. ${ }^{3-5}$

As Table 1 demonstrates, researchers typically developed 3D scanners for measurement (geometry) or visualization (texture), using photogrammetry, lasers, or millimeter wave. Several scanners are now available (http://3dlinks.com and http://simple3d.com).

\section{Scanning technologies}

Three-dimensional body scanners employ several technologies, including $2 \mathrm{D}$ video silhouette images, whitelight phase measurement, laser-based scanning, and radio-wave linear arrays, as Figure 2 illustrates.

Photogrammetry. These 3D scanners project patterns of light on the body. A video projector produces the pattern and the cameras record the distorted pattern. Manufacturers of this type of scanner include $\mathrm{TC}^{2}$, InSpeck, and Wicks and Wilson.

Laser scanning. This type of 3D scanner uses harmless, invisible lasers to measure the body. They work on the principle of triangulation, projecting a single point, line, or multiple lines onto the subject, and using a video camera offset from the laser source to view the laser light on the subject being scanned. Cyberware, Hamamatsu Photonics, and Human Solutions all manufacture laser scanners.

Millimeter wave. These 3D scanners use linear-array radio-wave technology to scan a subject's body, and they can scan through clothing. Intellifit is one company that currently offers a radio-wave scanner.

Scanners projecting light patterns are based on conventional 2D cameras. They usually feature several pairs 


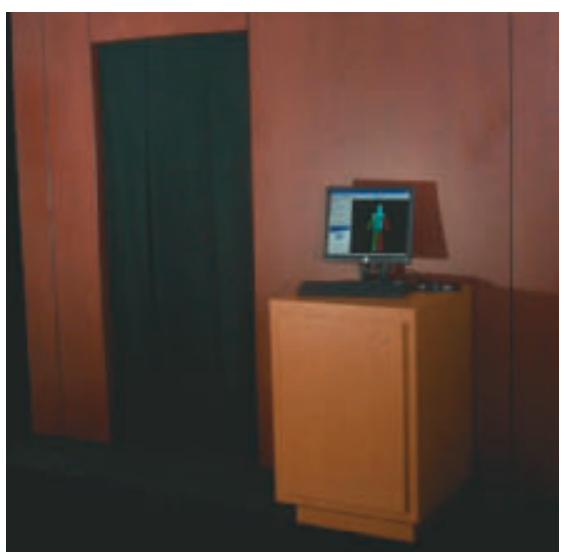

(a)

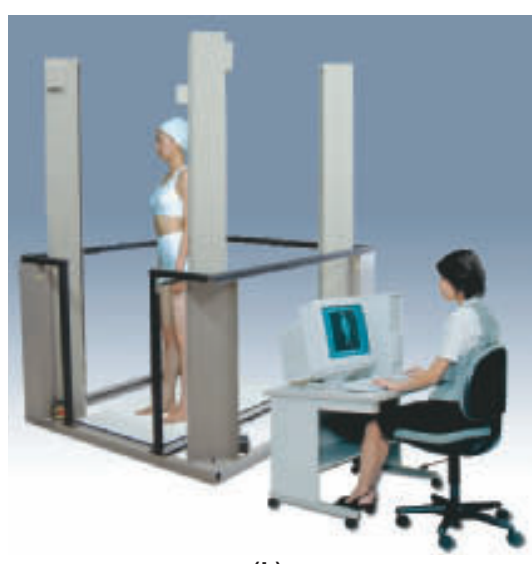

(b)

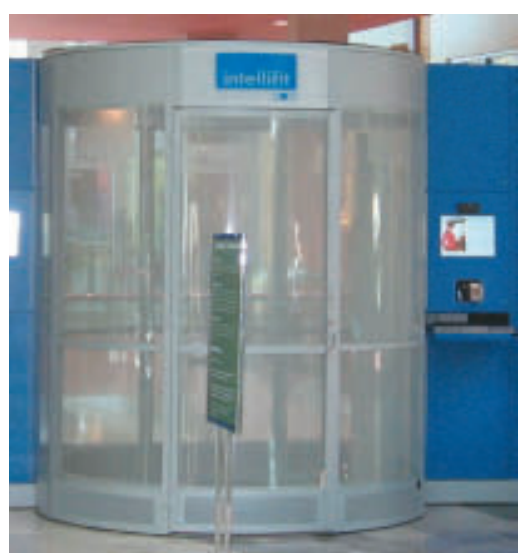

(c)

Figure 2. Body scanners. (a) The TC² photogrammetry scanner, (b) Hamamatsu laser scanner, and (c) Intellifit radio-wave scanner are examples of scanning technologies.

of cameras, with the images sent to a computer that calculates the binocular disparities between corresponding points in each pair of images. The 3D geometry is inferred using stereoscopy. In some models, the scanner projects a sinusoidal pattern on the object to generate a rich texture that helps identify feature points.

Laser range scanners are based on an array of depth sensors such as the one Chiyoharu Horiguchi ${ }^{5}$ developed for the Hamamatsu body line scanner range. These sensors fire a laser beam at the object and measure the direction of the reflected ray to calculate the distance between the sensor lens and the incident surface. With the location and orientation of the sensor known from the scanner's construction and calibration, it's easy to calculate the Cartesian coordinates of the point in space.

Millimeter-wave-based scanners, such as the one Intellifit produces, send a safe, low-power radio wave toward a person's fully clothed body. The vertical wand in the Intellifit system contains 196 small antennas that send and receive low-power radio waves. In the $10 \mathrm{sec}-$ onds it takes for the wand to rotate around a clothed person, the radio waves send and receive low-power signals. The signals don't "see" the person's clothing, but reflect off the skin, which is basically water.

Here, we're principally concerned with 3D wholebody scanners, most of which were designed for measurement capture (geometry) rather than visualization (texture). However, with the growing interest in healthcare visualization (for example, identifying melanomas or performing reconstructive and cosmetic surgery), an increasing number of high-resolution color scanners, such as $3 \mathrm{dMD}$ and Canfield, use passive and active stereo photogrammetry.

\section{D human-body modeling}

In $3 \mathrm{D}$ whole-body scanning, body modeling begins with conversion of the point-cloud surface representa- tion to a parametric form to facilitate both the extraction of measurements and visualization to describe surface features. The successful location of body landmarks is key to the reliable automated extraction of (linear) measurements from 3D body scans.

Maria C. Ruiz and her colleagues ${ }^{6}$ developed a suite of 3D shape-analysis tools for the UK National Sizing Survey (SizeUK). ${ }^{1}$ The tools calculate several attributes characterizing the shape of each torso while they create these canonical representations. The representations are stored in the database together with a summary of their statistical properties over all male and all female subjects.

The attributes allow using software tools to search the database efficiently to compare, analyze, and view averaged scans within a user-selected range of body shapes.

\section{HEALTH AND WELL-BEING APPLICATIONS}

Just as we can broadly divide 3D scanning systems into measurements (geometry) and visualization (texture), we also can subdivide healthcare applications into measurement (size, shape, surface area, volume) and visualization (head, chest, whole body), as Table 2 illustrates.

For our review, we've divided healthcare applications into four groups: epidemiology, diagnosis, treatment, and monitoring, although applications such as growth defects might appear in multiple cells.

\section{Epidemiology}

Epidemiology is the study of the distribution and determinants of health-related states or events in specified populations, and the application of this study to the control of health problems. For example, with the growing prevalence of obesity, especially among children, ${ }^{7}$ measurements of size and shape remain at the forefront of monitoring the population and screening individual subjects.

Anthropometric surveys. Large-scale anthropometric surveys have been fundamental to epidemiology for 
Table 2. 3D healthcare scanning application taxonomy.

\begin{tabular}{|c|c|c|c|c|}
\hline Application & Epidemiology & Diagnosis & Treatment & Monitoring \\
\hline \multicolumn{5}{|l|}{ Measurement } \\
\hline Size & Anthropometric surveys & Growth defects & Scoliosis & Fitness and diet \\
\hline Shape & Screening & Abdominal shape & Prosthetics & Obesity \\
\hline Surface area & & Lung volume & Drug dosage & Diabetes \\
\hline Volume & & & Burns & \\
\hline \multicolumn{5}{|l|}{ Visualization } \\
\hline Head & & Melanomas & Eating disorders & \\
\hline Chest & & & Facial reconstruction & \\
\hline Whole body & & & Cosmetic surgery & \\
\hline
\end{tabular}

over half a century. The recent adult sizing surveys the clothing industry commissioned in the United Kingdom and United States (SizeUK and SizeUSA $)^{1}$ illustrate the potential of 3 D body scanning for epidemiological surveys. SizeUK and SizeUSA each measured 11,000 subjects using 3D body scanners. The scanners automatically extracted 130 body measurements from each subject, leaving only 8-10 measurements to be handtaken for such things as height and head girth, and special measurements of the hands and feet. The survey information included personal details on each of the 11,000 adult subjects (excluding name and address), 140 measurements per subject (or 1.5 million measurements in total), 22,000 point clouds, and 40 market-research and lifestyle questions per subject.

This information yielded a 24,000-page report that included a statistical analysis of information for each measurement covering mean, maximum/minimum, and frequency (in total or cut by age, region, socioeconomic group, or ethnicity); a 3D shape analysis showing models of selected population subsets (also provided in total or cut by age, region, socioeconomic group, or ethnicity); and cross-correlation charts of measurements and 3D shape. Figure 3 illustrates the shape variation of size 12 women in the SizeUK survey.

Further applications. Due to its noninvasiveness and ease of use, 3D body scanning is ideal for screening large populations of subjects, particularly to simultaneously screen for multiple conditions. Although the BMI is used to classify obesity, it can't distinguish fat and lean tissue. Likewise, scoliosis is usually screened using the Adams forward-bend test, where the two sides of the torso are visually compared at the thoracic and thoracolumbar level. However, some have questioned this test's value, as it fails to identify a proportion of children who test positive using a scoliometer. Alfred Schmitz and his colleagues $^{8}$ reported that laser scanning provided a rapid and accurate measurement, with automatic calculation of the deformity sufficiently accurate for most purposes.

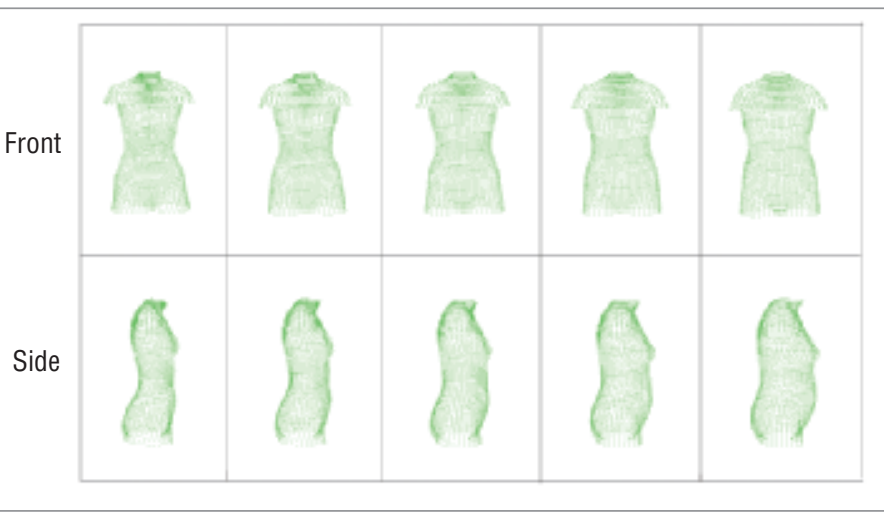

Figure 3. Body-shape variation. SizeUK software shows the shape variation of size 12 women in the United Kingdom.

While 3D scanning should be suitable for screening children for obesity and growth defects, because subjects must undress to their underwear, certain ethics and data-protection issues must be addressed.

\section{Diagnosis}

Two applications are highlighted for diagnoses. Measurement helps detect deformities, while visualization assists in skin analysis.

Deformity detection. A deformity or growth defect is defined as an affliction in which some part of the body is misshapen or malformed. Three-dimensional scanning offers the capacity to improve diagnosis of a wide range of body deformities that arise from genetic constitution, trauma, pathology, or occupation.

Skin analysis. Analysis using sophisticated image-processing software can evaluate skin conditions ranging from wrinkles and porphyrins (bacteria in pores that can lead to acne) to melanomas, the most serious and least common form of skin cancer.

Automated complexion analysis is rapidly gaining popularity as a tool for managing patients at high risk for melanoma. Many of the leading cancer centers and dermatology programs in the US employ this technique to aid in the early detection of suspicious lesions. An 


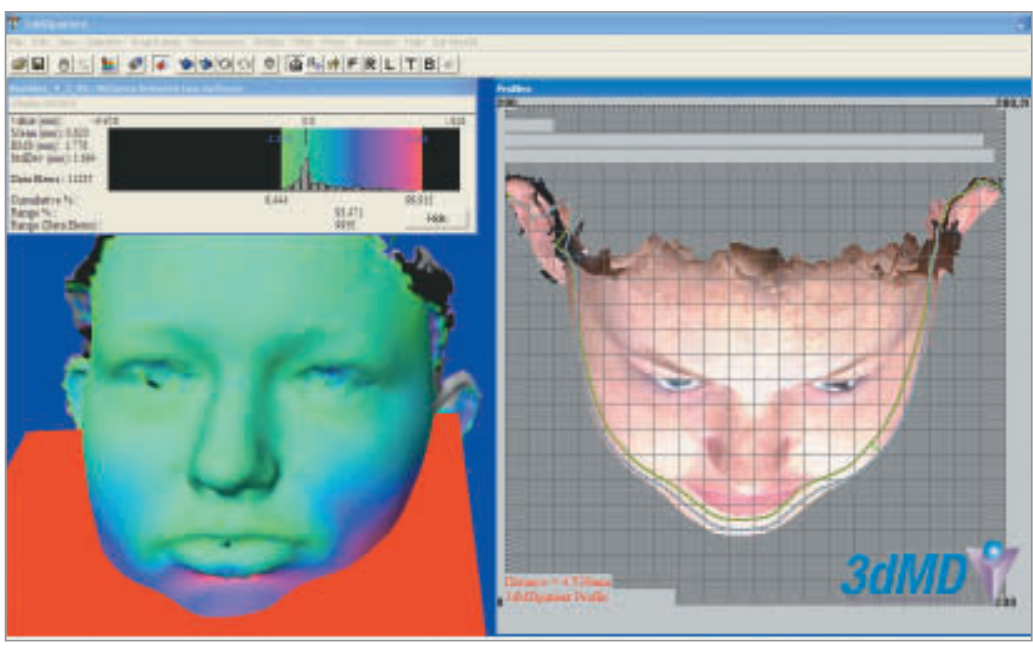

Figure 4. Visualization. The 3dMDpatient software platform provides medical and dental professionals with advanced landmarking and measurement-calculation systems, 3D facial analytic modules, and angle measurement.

example is the Canfield Visia complexion-analysis system, which couples a Canfield facial-imaging booth with Procter \& Gamble Visia analysis software.

The facial-imaging booth (not strictly a scanner) captures digital photos of the face. The Visia system software then analyzes wrinkles, evenness (color variation in the skin tone), pores, pigmentation, porphyrins, and the ultraviolet spots that are characteristic of photodamage, typically from overexposure to sun.

Further applications. Medical professionals can use a 3D body scan to obtain several other diagnoses, such as lung function, estimation of bleeding in hemophilia, and abdominal-shape characteristics that might act as a marker for the metabolic syndrome. ${ }^{9}$ Lung function is difficult to test for, especially in younger age groups. Volumetric analyses by 3D scanning is simple to perform, and, unlike direct-measurement methods such as spirometry, won't influence the trait being measured.

\section{Treatment}

When considering clinical treatment, a single 3D scan provides 2D linear measurements (waist, hips, and ratios), 3D body shape (chest, stomach), skin-surface area (whole body, body part), and volume. For example, clinicians use skin surface-area measurements for burn treatment and to calculate drug and chemotherapy doses for use in many circumstances. It's also the preferred criterion for indexing the glomerular filtration rate. ${ }^{10}$

Burn treatment. Calculating the total body-surface area of a burn is critical to estimating the amount of fluid needed to resuscitate patients as the Parkland formula specifies.

To date, to estimate the total body-surface area, physicians have relied on a clinical estimation per rule of nines, which assumes that the palm represents 1 percent of the total body-surface area and requires guessing the approximate number of palms it would take to cover the burn. The burned surfaces are recorded on a 2D Lund and Browder chart. Any inaccuracies can lead to underresuscitation, resulting in possible renal failure or massive fluid overload, pulmonary edema, and death.

Medical-scanning companies such as 3dMD have developed burn-application software to allow the user to display $3 \mathrm{D}$ surfaces, mark up burns by degree, and take measurements from them. The total burned body-surface area is then calculated and automatically entered into the Parkland formula for estimation of resuscitation fluids.

Comparisons of total body-surface area calculated with $3 \mathrm{dMD}$ software and using the Lund and Browder method produced a scanner approach that's 98 percent accurate, the company claims, compared with the 70 percent accuracy produced with the Lund and Browder method alone (http://asps.confex.com/asps/2003am/techprogram/ paper_3985.htm). Figure 4 depicts the 3dMDpatient software platform, which has advanced measurement capabilities and allows medical professionals to perform preoperative and postoperative comparisons.

Further applications. Three-dimensional scanning integrates with numerous aspects of treatment: accurate prescription of medicines, craniofacial surgery, orthotics and prosthetics, and treatment of eating disorders.

For drug and chemotherapy dosages, the measurement of surface area will likely prove particularly important. Due to differences in physiology, children can't be considered "little adults"; however, the appropriate calculation of pediatric drug dosages is a notoriously neglected area, with potentially serious consequences for patient outcome. Three-dimensional scanning facilitates measuring this important body parameter.

For eating disorders such as anorexia nervosa, where distorted body image is a defining characteristic, normalization of body image is critical for recovery. Presenting objective information about body shape can reduce bodyimage distortion, so that patients view their body shapes more realistically, while cognitive-behavioral methods have achieved reductions in body-image disturbance.

\section{Monitoring}

Because it's noninvasive, 3D body scanning is also useful for monitoring longitudinal changes in body morphology, whether due to exercise, nutrition, or diet programs administered as part of clinical treatment or through attendance at a gym or diet club. Medical personnel can overlay repeated scans of a subject to show changes in body shape over time, which is a great motivator to continue the program, from treating clinical 
obesity or sculpting the body to improve appearance.

Exercise and diet. Most people commonly use weighing scales and the mirror to track well-being. Arguably, people are most interested in their body's appearance, where they have gained muscle or lost fat. Hence, fitness and diet centers are an obvious market for 3D body scanners, and a few pioneering systems are already in use in gyms.

A case study is Wicks and Wilson (www.wwl.co.uk), which provides body visualization, measurement, and tracking infrastructure to the

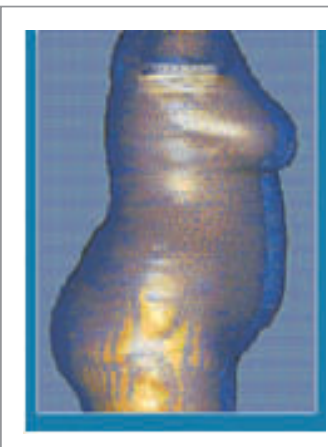

(a)
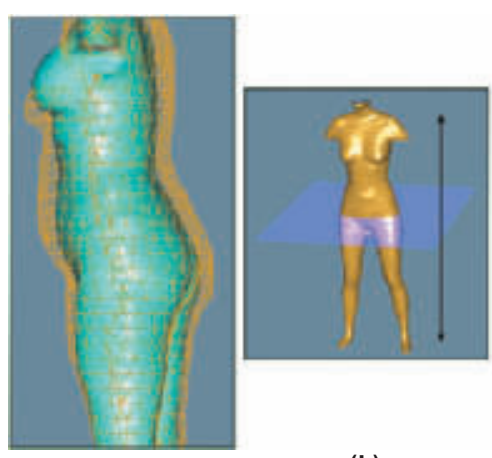

(b)

Figure 5. Monitoring body changes. Overlapping images (a) and cross-section measurements (b) can be used to monitor changes in body shape.

health and fitness market. The user gets an objective 3D body view from a single scan. This can be used to assess body symmetry and muscle imbalance and to analyze posture, such as arching of the lower back and roundedness of the shoulders. The software also provides girth crosssection measurements (thousands of slices through the body) at the chest, waist, and hips.

Over time, with multiple scans, the user can overlay images to track changes in shape and posture. As Figure 5 illustrates, the "new" body can be overlapped with the "old" one so the user can see exactly where the changes have occurred.

Further applications. Managing obesity by modifying dietary intake or activity level or with pharmacological or psychological intervention requires follow-up to assess the degree of weight loss. Obesity treatment is aimed at reducing harmful visceral fat, rather than body weight per se. Increases in muscle mass, or weight maintenance in growing children, are seen as acceptable effects of treatment, especially where professionals have advocated exercise.

Medical professionals can adapt 3D scanning to highlight those areas of surface shape that have changed within individuals over time, letting the clinician monitor treatment efficacy (for example, abdominal shape) while also engaging patient interest and hence increasing patient motivation.

T he time is ripe for a grade shift in the use of scanning technology, exploiting the potential of whole-body scanners for routine clinical practice in the same way that other techniques, such as MRI, $\mathrm{x}$-ray, and CT scanning have revolutionized imaging of the internal body. Three-dimensional body-surface scanning is poised to become a mainstream medical tool of major value. Now that appropriate hardware is available, the limiting factor is the software, which is rapidly becoming more sophisticated.

As with any new healthcare technology, medical professionals must consider several ethics, privacy, and

data-protection issues. With patients being scanned in their underwear and 3D scanners producing higher-resolution images, medical professionals must demonstrate sensitivity when using scans and showing them to patients, especially adolescents and those with eating disorders. However, the enormous potential benefits of $3 \mathrm{D}$ body scanning outweigh most of these issues.

\section{Acknowledgments}

We thank Hamamatsu Photonics, which stimulated UCL's work in 3D body scanning and funded our early research, and our colleagues Bernard Buxton, Laura Dekker, Ioannis Douros, and Tony Ruto. We also thank David Bruner and our collaborators at $\mathrm{TC}^{2}$ who worked closely with us on conducting the UK National Sizing Survey (SizeUK).

\section{References}

1. P.C. Treleaven, “Sizing Us Up," IEEE Spectrum, Apr. 2004, pp. 28-31.

2. P.R.M. Jones et al., "Three-Dimensional Surface Anthropometry: Applications to the Human Body," Optics and Lasers in Eng., Sept. 1997, pp. 89-117.

3. P.R.M. Jones, K. Brooke-Wavell, and G. West, "Format for Human Body Modelling from 3D Body Scanning," Int'l J. Clothing Science Technology, Mar. 1995, pp. 7-16.

4. M.H. Demers et al., "Three-Dimensional Surface Capture for Body Measurement Using Projected Sinusoidal Patterns," Proc. SPIE, Mar. 1997, pp.13-25.

5. C. Horiguchi, "BL (Body Line) Scanner: The Development of a New 3D Measurement and Reconstruction System," Int'l Archive Photogrammetry and Remote Sensing, vol. 32, 1998, pp. 421-429.

6. M.C. Ruiz et al., "Web-Based Software Tools for 3D Body Database Access and Shape Analysis," Proc Numerisation 3D Scanning, 2002; www.cs.ucl.ac.uk/staff/m.ruiz/ruiz_buxton_ douros_treleaven_scanning2002.pdf. 
7. C. Walton et al., "Body Fat Distribution, Rather than Overall Adiposity, Influences Serum Lipids and Lipoproteins in Healthy Men Independently of Age," Am. J. Medicine, Nov. 1995, pp. 459-464.

8. A. Schmitz et al., "Anthropometric 3D-Body Scanning in Idiopathic Scoliosis," Z Orthop Ihre Grenzgeb, Nov./Dec. 2002, pp. 632-636.

9. J.D. Lin et al., "Comparison of Three-Dimensional Anthropometric Body Surface Scanning to Waist-Hip Ratio and Body Mass Index in Correlation with Metabolic Risk Factors," J. Clinical Epidemiology, Aug. 2002, pp. 757-766.

10. C.Y. Yu, Y.H. Lo, and W.K. Chiou, "The 3D Scanner for Measuring Body Surface Area: A Simplified Calculation in the Chinese Adult," Applied Ergonomics, May 2003, pp. 273-278.
Philip Treleaven is pro-provost and professor of computing at University College London and director of the UK National Sizing Survey (SizeUK). His research interests include 3D body scanning and intelligent systems. Treleaven received a $\mathrm{PhD}$ in computer science from the University of Manchester. He is a member of the IEEE and the IEEE Computer Society. Contact him atp.treleaven@cs.ucl.ac.uk.

Jonathan Wells is a reader in pediatric nutrition at University College London's Institute of Child Health. His research interests include the measurement of body composition and energetics in healthy children and patients. He received a $\mathrm{PhD}$ in biological anthropology from the University of Cambridge.Contact himatj.wells@ich.ucl.ac.uk.

\section{Want to win a free trip to SC07?}

Francis Sullivan (IDA Center for Compuling Sciences) has a challenge for you...

\section{What w would Y YOU cro compule?}

"A lot of my working life has been spent arguing for more compule power and explaining the wonderful things that will happen when a more powerful machine is delivered. So far, nobody has called my bluff by saying, 'Okay, you can have all the compuling you want. Now, what are you going to do with it?'

Lel's fontasize. Suppose you had available, at your desk, a pelaflop machine. Assume that power and cooling were somehow taken care of and that you could get the full power of the machine by writing programs in a flexible and expressive language, no heroics necessary."

Email your answer in a 1,000-word essay to Jenny Stout [jstout@computer.org], with the subject line "petaflop challenge" by IO August 2007. The winner will receive a year's subscriplion to Compuling in Science \& Engineering magazine and will see their entry published in the

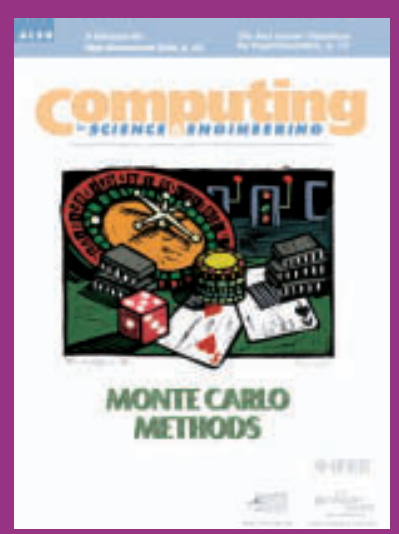
magazine's November/December 2007 issue. SC07 will also cover the winner's travel costs to attend its conference in Reno, Nevada, USA. SCO7 is the internalional conference for high-performance compuling, networking, storage and analysis. Visit hitp://sc07.supercompuling.org for conference details. 